\title{
Estrategias de financiamiento aplicadas por empresas del sector petroquímico en un entorno competitivo
}

\author{
Financing strategies applied by companies of the petrochemical sector in a \\ competitive environment
}

\section{Estratégias de financiamento aplicadas por empresas do setor petroquímico em ambiente competitivo}

Recibido: enero 2020

Arbitrado: febrero 2020

Publicado: mayo 2020

\section{ABSTRACT}

The changes in the business

Los cambios en el entorno empresarial experimentados a escala mundial en las últimas décadas, demandan administrar y manejar el dinero que necesitan para operar. Atendiendo a estas consideraciones, surge el presente artículo cuyo propósito es identificar las estrategias de financiamiento aplicadas por empresas del sector petroquímico. La investigación fue descriptiva, de diseño no experimental, transeccional y de campo. La población estuvo conformada por seis (6) empresas, vigentes y en ejecución dentro del complejo petroquímico Ana María Campo. Se realizaron entrevistas a veintidós (22) gerentes de dichas empresas. Los resultados indican que las formas de financiamiento a corto plazo son las que ocupan mayor peso dentro de las estrategias financieras que optan para la obtención de sus recursos operativos así como de inversión. Adicional a esto, se pudo detectar que en las estrategias de financiamiento en el largo plazo son consideradas las fuentes internas como base de este tipo de financiamiento.

Palabras clave: Estrategias de financiamiento; financiamiento a corto plazo; financiamiento a largo plazo; sector petroquímico, tipo de financiamiento environment experienced on a global scale in recent decades require managing and managing the money they need to operate. Taking into account these considerations, this article arises whose purpose is to identify the financing strategies applied by companies in the petrochemical sector. The research was descriptive, non-experimental, transectional and field design. The population was made up of six (6) companies, current and in execution within the Ana María Campo petrochemical complex. Twenty-two (22) managers of said companies were interviewed. The results indicate that the forms of short-term financing are those that occupy the greatest weight within the financial strategies that they choose to obtain their operating resources as well as investment. In addition to this, it was detected that in long-term financing strategies, internal sources are considered as the basis for this type of financing.

Key words: Financing strategies; short-term financing; long-term financing; petrochemical sector, type of financing 《regoria Nava

goganava71@gmail.com

ORCID: 0000-0002-6765-4928

Universidad del Zulia, Núcleo Costa Oriental del Lago, Venezuela
RESUMO

As mudanças no ambiente de negócios experimentadas em escala global nas últimas décadas exigem gerenciar e administrar o dinheiro de que precisam para operar. Levando em conta essas considerações, surge este artigo que tem por objetivo identificar as estratégias de financiamento aplicadas por empresas do setor petroquímico. A pesquisa foi descritiva, não experimental, transversal e de campo. A população era composta por seis (6) empresas, atuais e em execução no complexo petroquímico Ana María Campo. Foram entrevistados vinte e dois (22) gestores das referidas empresas. Os resultados indicam que as formas de financiamento de curto prazo são as que ocupam maior peso dentro das estratégias financeiras que optam para obter seus recursos operacionais e também de investimento. Além disso, detectou-se que nas estratégias de financiamento de longo prazo, as fontes internas são consideradas como base para este tipo de financiamento.

Palavras-chave: Estratégias de financiamento; financiamento de curto prazo; financiamento de longo prazo; setor petroquímico, tipo de financiamento 


\section{INTRODUCCIÓN}

$\mathrm{E}$ I nuevo escenario que plantea la economía mundial y el incremento de la competencia implican retos para el mundo empresarial venezolano, especialmente en el sector petroquímico, el cual se ha visto afectado en los últimos años por la incursión de empresas internacionales. En efecto, algunas empresas a nivel nacional han experimentado procesos de modernización y reorganización o han adoptado estrategias para la reducción de costos de distribución y aprovisionamiento, como manera de responder a las exigencias del entorno.

En este marco de referencia, varios autores, en los conceptos y teorías aportados, hacen referencia a la palabra estrategia definiéndola como metas u objetivos a alcanzar por parte de las empresas para lograr su misión. Chandler (2010), precisa que estrategia es determinar los objetivos y las metas fundamentales a largo plazo, adoptar políticas correspondientes y asegurar los recursos necesarios para llegar a esas metas.

De igual manera, Francés (2006), afirma que una estrategia bien formulada permite canalizar los esfuerzos y asignar los recursos de una organización, y la lleva a adoptar una posición singular y viable, basada en sus capacidades internas (fortalezas y debilidades), anticipando los cambios en el entorno, los posibles movimientos del mercado y las acciones de sus competidores (oportunidades y amenazas).

Partiendo de estas ideas, se resalta que en Venezuela las empresas que prestan servicio a la industria nacional, se desenvuelven en una serie de problemas que en la mayoría de los casos no logran superar; lo que conlleva hacia el fracaso seguro que tiende a complicarse bajo la presencia de ciertos elementos que obstaculizan no solo su crecimiento sino también su desarrollo; por lo cual deben estar en evaluación continua de todas sus estrategias.

Frente a estas circunstancias, estas empresas requieren formular estrategias que permitan el acceso a diversas fuentes de financiamiento disponibles en el mercado, con el fin de cubrir sus objetivos financieros y tomar decisiones de inversión acertadas, que les ayuden a consolidarse y enfrentar el desafío de un mayor crecimiento dentro de un contexto de apertura económica.

En consecuencia, las empresas requieren contar con una adecuada gerencia financiera que le permita manejar las diferentes fuentes de recursos a las que pueden acceder para llevar a cabo de la manera más conveniente sus actividades y financiar sus procesos de expansión, mantener su participación en el mercado y adecuarse a los niveles de competitividad que exige cualquier sector en Venezuela. Principalmente porque en ausencia de un mercado de capitales desarrollado las posibilidades de financiamiento se concentran en la reinversión de utilidades retenidas y aplicación de reservas, emisión privada de acciones, créditos bancarios, créditos de entes gubernamentales, integración vertical en redes de proveedores y pasivos laborales.

Atendiendo a estas consideraciones se insertan las empresas arraigadas en la Costa Oriental de Lago, específicamente en el municipio Miranda, prestadoras de servicios a la industria petroquímica nacional y sus filiares, las cuales requieren conocer mecanismos empresariales que les permitan 
enfrentar las condiciones de incertidumbre por las que normalmente transitan, brindándoles aportes que contribuyan, de alguna forma, a orientar la administración de sus finanzas, y por ende, el incremento de su rentabilidad.

En este sentido, el objetivo del presente artículo radica en identificar las estrategias de financiamiento aplicadas por empresas del sector petroquímico, específicamente las del municipio Miranda, para conocer como administran sus finanzas, para brindar información pertinente que permita orientar su gestión económica, manteniendo un nivel de actividad adecuado encaminado al cumplimiento de sus objetivos.

\section{MÉTODO}

$\mathrm{E}$ I tipo de investigación en el estudio, se calificó como descriptiva, de diseño no experimental, transeccional y de campo, ya que se analizó la variable estrategias de financiamiento aplicadas por empresas del sector petroquímico. Además los datos se recolectaron directamente en el lugar donde se desenvuelve la variable objeto de estudio, sin manipulación y en un solo momento determinado.

La población estuvo constituida por las seis empresas que prestan servicio en la ejecución de proyectos de ingeniera, vigentes y en ejecución, en el complejo petroquímico Ana María Campo, cuyas unidades informantes estuvo conformado por el nivel gerencial, a quienes se les aplico un cuestionario con escala de frecuencia

En este sentido, el instrumento fue valorado por cinco expertos en el área de la variable a investigar en el estudio. Por otra parte el instrumento fue sometido a una prueba pilo para calcular su confiabilidad a través del coeficiente Alfa de Cronbach, cuyo valor fue 0,89. Finalmente, para el análisis de las frecuencias la investigadora consideró conveniente fijar un baremo de interpretación, el cual se recoge en el cuadro 1.

Cuadro 1. Interpretación del porcentaje de la distribución de frecuencia

\begin{tabular}{ccl}
\hline RANGO & CATEGORÍA & \multicolumn{1}{c}{ INTERPRETACIÓN } \\
\hline $\mathrm{S}+\mathrm{CS} \geq 70 \%$ & $\begin{array}{c}\text { Alta aplicación } \\
\text { Alta fortaleza }\end{array}$ & $\begin{array}{l}\text { Alta aplicación del indicador, dimensión o variable. } \\
\text { Implica alta fortaleza para las empresas }\end{array}$ \\
$40 \% \leq \mathrm{S}+\mathrm{CS}<70 \%$ & $\begin{array}{c}\text { Moderada aplicación } \\
\text { Leve fortaleza }\end{array}$ & $\begin{array}{l}\text { Moderada aplicación del indicador, dimensión o } \\
\text { variable. } \\
\text { Implica leve fortaleza para las empresas }\end{array}$ \\
$\mathrm{S}+\mathrm{CS}<40 \%$ & $\begin{array}{c}\text { Baja aplicación } \\
\text { Debilidad }\end{array}$ & $\begin{array}{l}\text { Baja aplicación del indicador, dimensión o variable. } \\
\text { Implica debilidad para las empresas }\end{array}$ \\
\hline
\end{tabular}




\section{RESULTADOS}

$\mathrm{E}$ I análisis de los resultados ha sido estructurado en tablas contentivas de toda la información recogida sobre la variable, clasificada por dimensión, indicador e ítem. Por consiguiente, al hacer referencia al objetivo orientado a identificar las estrategias de financiamiento aplicadas en las empresas del sector petroquímico, se logró obtener la información necesaria para mostrar estos resultados.

Vale recordar que este objetivo se alcanza a través del análisis de las estrategias de financiamiento, la cual fue dividida en dos dimensiones: estrategias de financiamiento a corto plazo y estrategias de financiamiento a largo plazo. Comenzando el análisis de la primera dimensión, cuyos indicadores son: crédito comercial o crédito de proveedores, línea de crédito a corto plazo, financiamiento de cuentas por cobrar y financiamiento por inventarios, se presentan las siguientes tablas para el primer indicador señalado:

De acuerdo a los valores obtenidos, como puede observarse en la tabla 1, un 95,45\% en promedio de los encuestados respondieron a los ítems involucrados en las alternativas siempre y casi siempre, indicando que el crédito comercial o crédito de proveedores como estrategia de financiamiento en el corto plazo tiene muy alta aplicación, esto implica alta fortaleza para las empresas del sector bajo estudio.

Tabla 1. Crédito comercial o crédito de proveedores.

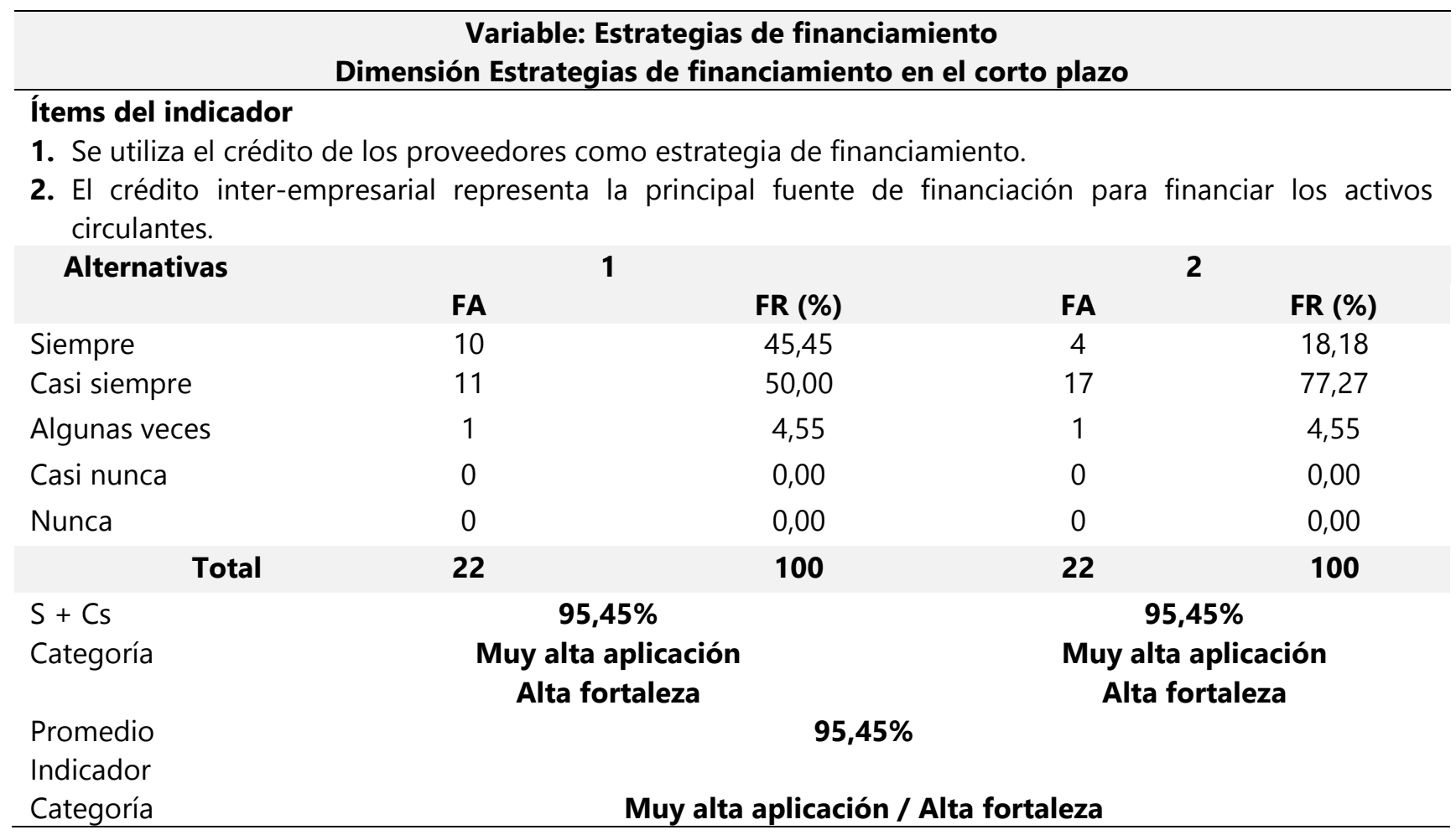


Asimismo, se observa para ambos ítems que el 95,45\% de los encuestados respondieron en las opciones positivas $(S+C s)$, indicando que se tiene muy alta aplicación de que se utilice el crédito de los proveedores como estrategia de financiamiento $y$, el crédito inter-empresarial represente la principal fuente de financiación para financiar los activos circulantes, confiriéndoles alta fortaleza a las empresas en estos dos aspectos.

Lo mostrado coincide de alta manera con lo expuesto por Brachfield (2009), quien opina que muchas empresas nunca hubieran podido iniciar sus actividades sin el crédito concedido por sus proveedores; agrega que el crédito inter-empresarial representa la principal fuente de financiación para las empresas que no pueden acceder al crédito bancario para financiar sus activos circulantes. De modo que el crédito comercial o crédito de proveedores constituye la principal fuente espontánea de financiación que disponen las empresas y es una de las fuentes más flexibles a corto plazo.

Dado los resultados, se evidenció que para las empresas del sector petroquímico el crédito comercial o crédito de proveedores constituye uno de sus principales fuentes de financiamiento a corto plazo, tanto en la modalidad de crédito de los proveedores como del crédito interempresarial, siendo esto común en el caso de las empresas pequeñas, debido a que éstas frecuentemente no califican para ser financiadas por otras fuentes de financiamiento, debiendo utilizar el crédito comercial.

En lo concerniente al indicador línea de crédito a corto plazo, se observa, en la tabla 2, un promedio de frecuencias de $88,64 \%$, indicando muy alta aplicación de este indicador como estrategia de financiamiento en el corto plazo en las empresas analizadas, implicando alta fortaleza, según el baremo diseñado. Así, en el detalle de los ítems, el instrumento diseñado arrojó que el $90,91 \%$ de los encuestados señalan que siempre y casi siempre solicitan líneas de crédito a fin de cumplir de forma más holgada los compromisos; y el 86,36\% considera que siempre y casi siempre se financian a través de instituciones financieras, otorgándole alta fortaleza a las empresas bajo estudio, en lo que respecta a estos aspectos. 
Tabla 2. Línea de crédito a corto plazo.

\begin{tabular}{|c|c|c|c|c|}
\hline \multicolumn{5}{|c|}{$\begin{array}{c}\text { Variable: Estrategias de financiamiento } \\
\text { Dimensión Estrategias de financiamiento en el corto plazo }\end{array}$} \\
\hline \multirow{2}{*}{\multicolumn{5}{|c|}{$\begin{array}{l}\text { Ítems del indicador } \\
\text { 3. Solicitan líneas de crédito a fin de cumplir de forma más holgada los compromisos. } \\
\text { 4. Se caracteriza por financiarse a través de instituciones financieras. }\end{array}$}} \\
\hline & & & & \\
\hline \multirow[t]{2}{*}{ Alternativas } & \multicolumn{2}{|c|}{3} & \multicolumn{2}{|c|}{4} \\
\hline & FA & FR (\%) & FA & FR (\%) \\
\hline Siempre & 5 & 22,73 & 12 & 54,55 \\
\hline Casi siempre & 15 & 68,18 & 7 & 31,82 \\
\hline Algunas veces & 2 & 9,09 & 3 & 13,64 \\
\hline Casi nunca & 0 & 0,00 & 0 & 0,00 \\
\hline \multirow[t]{2}{*}{ Nunca } & 0 & 0,00 & 0 & 0,00 \\
\hline & 22 & 100 & 22 & 100 \\
\hline$S+C s$ & \multicolumn{2}{|c|}{$90,91 \%$} & \multicolumn{2}{|c|}{$86,36 \%$} \\
\hline \multirow[b]{2}{*}{ Categoría } & \multicolumn{2}{|c|}{ Muy alta aplicación } & \multicolumn{2}{|c|}{ Muy alta aplicación } \\
\hline & \multicolumn{2}{|c|}{ Alta fortaleza } & \multicolumn{2}{|c|}{ Alta fortaleza } \\
\hline \multicolumn{3}{|l|}{ Promedio Indicador } & \multicolumn{2}{|c|}{$88,64 \%$} \\
\hline Categoría & & Muy alta & $\mathbf{A l}$ & \\
\hline
\end{tabular}

Lo mostrado valida, en alta medida, lo postulado por Ross et al. (2006), para quienes este préstamo responde a necesidades de crédito a corto plazo para hacer compras estacionales, gasto de operación y capital de trabajo; las líneas de crédito permiten a las empresas pequeñas cumplir de forma más holgada los compromisos y, generalmente, este tipo de empresas se financian a través de instituciones financieras.

Así que, las empresas han considerado en muy alta medida la línea de crédito como una estrategia de financiamiento a corto plazo, han entendido las bondades que les permite este tipo de financiación para cubrir sus obligaciones de corto plazo, y el hecho de que apliquen este tipo de financiamiento pone en evidencia que han demostrado capacidad de liquidar la deuda ante las instituciones financieras.

El siguiente indicador que se analiza, en esta dimensión, es el financiamiento de cuentas por cobrar. Los resultados relativos a él, se resumen en la tabla 3, observándose que, en promedio, para el $95,45 \%$ de los encuestados a este tipo de financiamiento, siempre y casi siempre, se le otorga muy alta aplicación como estrategia de financiamiento a corto plazo, implicando alta fortaleza de la gestión financiera que llevan a cabo las empresas bajo estudio. 
Tabla 3. Financiamiento de cuentas por cobrar.

\section{Variable: Estrategias de financiamiento}

Dimensión Estrategias de financiamiento en el corto plazo

\section{Ítems del indicador}

5. Emplean la estrategia del financiamiento de cuentas por cobrar para obtener recursos que le permitan continuar su proceso productivo.

6. Conceden descuentos a sus clientes en caso de cancelar las deudas antes del plazo concedido.

\section{Alternativas}

Siempre
Casi siempre
Algunas veces
Casi nunca
Nunca
S + Cs
Categoría
Promedio Indicador
Categoría

5

FA

2 19

1

0

0 22

Total
Muy alta aplicación Alta fortaleza
FR (\%)

9,09

86,36

4,55

0,00

0,00

100

$95,45 \%$

\section{5,45\%}

Muy alta aplicación / Alta fortaleza
6

FA FR (\%)

\section{3}

13,64

18

81,82

$1 \quad 4,55$

$0 \quad 0,00$

$0 \quad 0,00$

$22 \quad 100$

$95,45 \%$

Muy alta aplicación

Alta fortaleza

Adicionalmente, la tabla muestra como para el 95,45\% de los encuestados, siempre y casi siempre, emplean la estrategia del financiamiento de cuentas por cobrar para obtener recursos que le permitan continuar su proceso productivo, y conceden descuentos a sus clientes en caso de cancelar las deudas antes del plazo concedido, asignándole a estas actividades la categoría de muy alta aplicación, constituyéndolas en altas fortalezas de las empresas estudiadas.

Los resultados validan lo planteado por Block y Hirt (2008), quienes afirman que las cuentas por cobrar son los activos de corto plazo valiosos y se deben comprometer de acuerdo a causas necesarias, como la obtención de recursos que le permitan continuar su proceso productivo, o para conceder descuentos a sus clientes en caso de cancelar las deudas antes del plazo concedido; ya que un deficiente uso puede excluir a la compañía de un préstamo a términos menos costoso que a través de un banco.

Visto así, se dedujo que las empresas analizadas actúan considerando en muy alta medida las posibilidades de financiarse en el corto plazo, de ahí que dentro de sus estrategias también aplican en muy alto grado la estrategia del financiamiento de cuentas por cobrar, considerándola como una opción para conseguir préstamos relacionados con el nivel de expansión del activo en cualquier punto del tiempo.

Ahora bien, en el orden de presentación que se lleva adelante, se tienen los resultados del último indicador considerado en la sub-dimensión estrategias de financiamiento en el corto plazo, referido al financiamiento por inventarios. En este sentido, en la tabla 4, se observa un promedio de 
frecuencias de 95,45\%, indicando muy alta aplicación de este indicador como parte de las estrategias de financiamiento en el corto plazo aplicadas por las empresas bajo estudio, lo cual implica alta fortaleza para las mismas.

Al desglosar los resultados por ítems, se evidencia para el ítem 7 como el $100 \%$ de los encuestados afirmaron que, siempre y casi siempre, emplean el financiamiento por medio de sus inventarios para obtener recursos que le permitan continuar su proceso productivo; y el 90,91\%, en el mismo sentido, afirman que usan el inventario como garantía de un préstamo, lo cual les confiere alta fortaleza dada una muy alta aplicación de estas actividades medidas por los ítems.

Los resultados validan a Gitman (2007), para quien el financiamiento por inventarios es aquel en el cual se usa el inventario como garantía de un préstamo, en que se confiere al acreedor el derecho de tomar posesión en caso de que la empresa deje de cumplir. Es importante ya que les permite a los directores de la empresa usan el inventario como fuente de recursos, gravándolo como colateral es posible obtener recursos de acuerdo con las formas específicas de financiamiento usuales, en estos casos, como son el almacenamiento de la fábrica, recibo de custodia, garantía flotante y la hipoteca.

Tabla 4. Financiamiento por inventarios.

\begin{tabular}{|c|c|c|c|c|}
\hline \multicolumn{5}{|c|}{$\begin{array}{c}\text { Variable: Estrategias de financiamiento } \\
\text { Dimensión Estrategias de financiamiento en el corto plazo }\end{array}$} \\
\hline \multicolumn{5}{|c|}{$\begin{array}{l}\text { Ítems del indicador } \\
\text { 7. Emplean el financiamiento por medio de sus inventarios para obtener recursos que le permitan } \\
\text { continuar su proceso productivo. } \\
\text { 8. Usan el inventario como garantía de un préstamo. }\end{array}$} \\
\hline \multirow[t]{2}{*}{ Alternativas } & \multicolumn{2}{|c|}{7} & \multicolumn{2}{|c|}{8} \\
\hline & FA & FR (\%) & FA & FR (\%) \\
\hline Siempre & 2 & 9,09 & 2 & 9,09 \\
\hline Casi siempre & 20 & 90,91 & 18 & 81,82 \\
\hline Algunas veces & 0 & 0,00 & 2 & 9,09 \\
\hline Casi nunca & 0 & 0,00 & 0 & 0,00 \\
\hline Nunca & 0 & 0,00 & 0 & 0,00 \\
\hline Total & 22 & 100 & 22 & 100 \\
\hline$S+C s$ & & $\begin{array}{l}\text { cación } \\
\text { eza }\end{array}$ & & ción \\
\hline \multicolumn{3}{|l|}{ Promedio Indicador } & \multicolumn{2}{|c|}{$95,45 \%$} \\
\hline Categoría & & Muy alta & Alta & \\
\hline
\end{tabular}


De esta manera, se dedujo que las empresas estudiadas han valorado este tipo de financiamiento en el corto plazo, aplicándolo para financiar situaciones vitales de sus actividades, como lo es el obtener recursos que le permitan continuar su proceso productivo, así como de garantía de un préstamo que les permita obtener dinero para resolver aspectos financieros en el corto plazo.

Luego de analizados los resultados para cada uno de los indicadores que conforman la dimensión estrategias de financiamiento en el corto plazo, en la tabla 5 se reflejan los resultados alcanzados para dicha dimensión. Se observa que la misma se ubica en la categoría de muy alta aplicación (93,75\%), indicando que en estas empresas la aplicación de sus estrategias de financiamiento en el corto plazo se constituye en alta fortaleza. Así, se observa que todos los indicadores se posicionaron en el rango donde la suma de las opciones siempre y casi siempre son: $75 \% \leq S+C S<100 \%$, indicando muy alta aplicación del indicador con implicación de alta fortaleza para las empresas.

Tabla 5. Estrategias de financiamiento en el corto plazo.

\begin{tabular}{lll}
\hline INDICADOR & S + Cs & CATEGORÍAS \\
\hline Crédito comercial o crédito de provedores & $95,45 \%$ & Muy alta aplicación / Alta fortaleza \\
Línea de crédito a corto plazo & $88,64 \%$ & Muy alta aplicación / Alta fortaleza \\
Financiamiento de cuentas por cobrar & $95,45 \%$ & Muy alta aplicación / Alta fortaleza \\
Financiamiento por inventarios & $95,45 \%$ & Muy alta aplicación / Alta fortaleza \\
\hline \multicolumn{1}{c}{ RESUMEN SUB-DIMENSIÓN } & $\mathbf{9 3 , 7 5 \%}$ & Muy alta aplicación / Alta fortaleza \\
\hline
\end{tabular}

Estos resultados son altamente coincidentes con lo manejado por Ross et al. (2006), en cuanto a que estas estrategias se concentran en las figuras de préstamos comerciales sin garantía como el crédito comercial o crédito de proveedores y línea de crédito; y préstamos con garantía, asumiendo el financiamiento de cuentas por cobrar y financiamiento por inventarios. De igual manera validan a Gitman (2007), cuando define el financiamiento a corto plazo como los adeudos que vencen al cabo de un año o menos, y son empleados para satisfacer necesidades estacionales y de activos circulantes. El financiamiento a corto plazo con garantía utiliza ciertos activos como garantía, así como el financiamiento a corto plazo sin garantía.

Visto estos resultados, de muy alta aplicación de las estrategias de financiamiento en el corto plazo, en las empresas bajo estudio, la estrategia de financiamiento a corto plazo, como parte de sus estrategias para financiar su actividad productiva, es una visión de la función financiera que debe desarrollar toda empresa según lo evidenciado en la teoría de este trabajo. Esta visión parte de las decisiones que influyen en los activos circulantes y pasivos circulantes, cuyos efectos deben reflejarse en la empresa en el lapso de un año.

Seguidamente se aborda el análisis de los indicadores de la segunda dimensión, denominada estrategias de financiamiento en el largo plazo, y que se corresponde a las estrategias de 
financiamiento. Cabe recordar, que la dimensión será estudiada a través de 4 indicadores: préstamos a largo plazo, capital propio, estrategias de dividendos y bonos. La tabla 6 muestra los resultados para el primer indicador: préstamos a largo plazo; donde se aprecia un promedio de frecuencias de 34,09\%, indicando baja aplicación del indicador como parte de las estrategias de financiamiento en el largo plazo, asociado a leve debilidad para las empresas bajo estudio.

Tabla 6. Préstamos a largo plazo.

Variable: Estrategias de financiamiento

Dimensión Estrategias de financiamiento en el largo plazo

Ítems del indicador

9. Solicitan financiamiento a largo plazo a instituciones financieras con el fin de asegurar el cumplimiento de sus actividades.

10. Utilizan los préstamos a largo plazo para proveer fondos por más de un año, de manera de aumentar las ganancias.

Alternativas

Siempre

Casi siempre

Algunas veces

Casi nunca

Nunca

Categoría

Promedio Indicador

Categoría
9

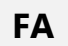

1

7

13

1

0

Total

22

$36,36 \%$

Baja aplicación

Leve debilidad
FR (\%)

4,55

31,82

59,09

4,55

0,00

100

n

34,09\%

Baja aplicación / Leve debilidad
10

FA

FR (\%)

4,55

27,27

63,64

4,55

0,00

100

$31,82 \%$

Baja aplicación

Leve debilidad

Como se puede apreciar en la tabla referida, todos los ítems muestran baja aplicación al arribar a frecuencias ubicadas entre el rango $25 \% \leq S+C S<50 \%$, indicando que en las empresas analizadas siempre y casi siempre se aplica de forma baja el que: soliciten financiamiento a largo plazo a instituciones financieras con el fin de asegurar el cumplimiento de sus actividades (36,36\%), y utilicen préstamos a largo plazo para proveer fondos por más de un año, de manera de aumentar las ganancias (31,82\%).

Así, no se alcanza validar a Gitman (2007), para quien los préstamos a largo plazo son usualmente un acuerdo formal para proveer fondos por más de un año y, la mayoría es para una mejora que beneficiará a la compañía de manera de aumentar las ganancias. Tampoco validan lo postulado por Van Horne y Wachowicz (2002), cuando señalan que, generalmente, los préstamos a largo plazo ocurren cuando está en perspectiva el aumento del capital, una inversión en activos o el comienzo de un negocio. 
Visto de esta forma, se dedujo que las empresas del sector petroquímico no están considerando los préstamos a largo plazo como una alternativa para financiar el crecimiento de su capacidad productiva en pro de aumentar sus ganancias, o de lograr aumentos de su capital, como tampoco inversiones en activos o nuevas líneas de negocio, por lo cual se les está confiriendo la categoría de leve debilidad.

La tabla 7 muestra los resultados para el indicador capital propio, observándose un promedio de frecuencias de 93,18\%, indicando que siempre y casi siempre, a criterio de los encuestados, el capital propio, como parte de las estrategias de financiamiento en el largo plazo que utilizan, se aplica con muy alta frecuencia, otorgándole la categoría de muy alta aplicación, implicando a su vez una alta fortaleza para dichas empresas.

Tabla 7. Capital propio.

Variable: Estrategias de financiamiento

Dimensión Estrategias de financiamiento en el largo plazo

\section{Ítems del indicador}

11. Utilizan préstamos de sus dueños como una fuente de financiamiento.

12. Se solicitan aportaciones de capital en acuerdos de la asamblea de accionistas.

\section{Alternativas}

Siempre

Casi siempre

Algunas veces

Casi nunca

Nunca

Categoría

Promedio Indicador

Categoría
11

FR (\%)

13,64

86,36

0,00

0,00

0,00

Total

22

100

$100 \%$

Muy alta aplicación

Alta fortaleza
12

FA

FR (\%)

50,00

36,36

13,64

0,00

0,00

100

$86,36 \%$

Muy alta aplicación

Alta fortaleza

$93,18 \%$

Muy alta aplicación / Alta fortaleza

De tal manera, se confirieron muy alta aplicación a las actividades medidas por los ítems utilizados, referidas a si utilizan préstamos de sus dueños como una fuente de financiamiento. (100\%), y solicitan aportaciones de capital en acuerdos de la asamblea de accionistas (86,36\%); implicando alta fortaleza para las empresas estudiadas en lo concerniente a estos aspectos.

Estos resultados coinciden, de forma alta, con lo expuesto por Ortiz (2005), para quien el financiamiento a largo plazo en las empresas a través del capital propio, pudiera estar representado por la utilización de préstamos de los dueños como una fuente de financiamiento, o por solicitud de aportaciones de capital en acuerdos de la asamblea de accionistas; cuyo objetivo 
sería aumentar la capacidad instalada, mejorar aspectos productivos, aumentar el capital de trabajo, inversiones en activos, creación de nuevas líneas de negocio, entre otras.

De acuerdo a lo evidenciado, se dedujo que en las empresas bajo estudio se le ha otorgado al capital propio un rol clave como fuente de financiamiento en el largo plazo, en donde se infiere que estas empresas al momento de requerir financiarse en periodos de pago superiores a un año consideran esta opción como una fuente segura, por lo cual los encuestados le han otorgado alta fortaleza en este aspecto.

Otro indicador considerado en la dimensión estrategias de financiamiento en el largo plazo, lo constituyen las estrategias de dividendos. La tabla 8 muestra los resultados obtenidos del trabajo de campo. Se observa que este indicador obtuvo una categoría de alta aplicación, al arribar a un promedio de frecuencias de 72,73\%, implicando leve fortaleza para las empresas estudiadas.

Estos resultados son reflejo de las actividades que se desarrollan en las empresas bajo estudio. En este sentido al consultarles si se autofinancian total o parcialmente en su actividad, reinvirtiendo las utilidades o excedentes que se generan, el 95,45\% consideró las opciones más positivas ( $+\mathrm{Cs}$ ) catalogada con muy alta aplicación e implicando alta fortaleza, según el baremo utilizado. No obstante, en el ítem 14, cuando se les pregunta si en caso de presentarse oportunidades de inversión que aseguren una rentabilidad convierten los dividendos en una fuente interna de financiamiento, el 50\% respondió casi siempre y el restante $50 \%$ algunas veces, indicando que esta actividad se realiza con alta aplicación convirtiéndose en una leve fortaleza para las empresas.

Las situaciones precedentes validan la posición de Vera (2009), para quien la empresa puede autofinanciarse total o parcialmente en su actividad reinvirtiendo las utilidades o excedentes que se generan año tras año; los cuales pertenecen a los accionistas comunes y deberían ser distribuidos a través del reparto de dividendos. En caso de presentarse oportunidades de inversión que asegure una rentabilidad y justifique su retención, éstos se convertirían en una fuente interna de financiamiento y se puede presentar en dos modalidades: utilidad no distribuida y dividendos en acciones. 
Tabla 8. Estrategias de dividendos.

\section{Variable: Estrategias de financiamiento}

Dimensión Estrategias de financiamiento en el largo plazo

\section{Ítems del indicador}

13. Se autofinancian total o parcialmente en su actividad, reinvirtiendo las utilidades o excedentes que se generan.

14. En caso de presentarse oportunidades de inversión que aseguren una rentabilidad convierten los dividendos en una fuente interna de financiamiento.

\section{Alternativas}

Siempre
Casi siempre
Algunas veces
Casi nunca
Nunca
S + Cs
Categoría
Promedio Indicador
Categoría

13

FA

10

11

1

0

0

22

Total

22
FR (\%)

45,45

50,00

4,55

0,00

0,00

100

$95,45 \%$

Muy alta aplicación

Alta fortaleza
14

FA

FR (\%)

0,00

50,00

50,00

0,00

0,00

22

100

\section{$50 \%$}

Alta aplicación

Leve fortaleza

$72,73 \%$

Alta aplicación / Leve fortaleza

De esta manera, las empresas del sector petroquímico analizado consideran como alternativa viable las estrategias de dividendos como fuente de financiamiento a largo plazo, basan el uso de las utilidades retenidas como forma de financiarse, es decir aquellos ingresos de la empresa que no se distribuyen en forma dividendos, por lo que la constituyen en una fuente de financiamiento interno.

Situación ésta que induce a pensar que las decisiones en torno a la utilización de los dividendos pudieran estar afectando significativamente los requerimientos de financiamiento externo de la empresa, de allí que en el préstamo a largo plazo muestren baja aplicación, puesto que si la empresa necesita financiamiento está optando por los dividendos no distribuidos y el uso del capital propio, disminuyendo la necesidad del financiamiento a obtenerse de manera externa (préstamos a largo plazo).

En este recorrido de los resultados, se tienen los obtenidos para el último indicador de la dimensión bajo estudio denominado bonos, los cuales se resumen en la tabla 9. Se evidencia que este indicador fue catalogado con baja aplicación, constituyéndose en leve debilidad para las empresas estudiadas, mostrado por un promedio de frecuencias relativas, en las opciones siempre y casi siempre, de $31,82 \%$. 
Tabla 9. Bonos.

Variable: Estrategias de financiamiento

Dimensión Estrategias de financiamiento en el largo plazo

\section{Ítems del indicador}

15. Utilizan bonos para recaudar cantidades importantes de fondos.

16. Emiten deuda a través de bonos para financiar proyectos de inversión

Alternativas

15

FA

Siempre

Casi siempre

Algunas veces

Casi nunca

Nunca

Total

$\mathbf{S}+\mathbf{C s}$

Categoría

Promedio Indicador

Categoría

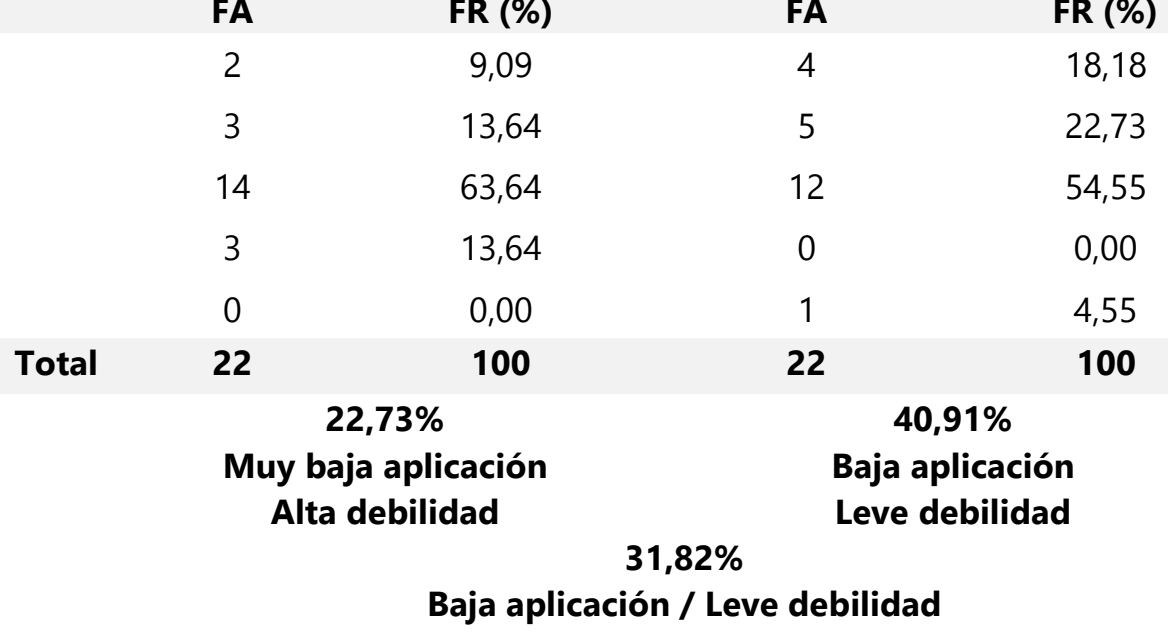

Al detalle se observa, para un $22,73 \%$ de los encuestados, siempre y casi siempre, se utilizan bonos para recaudar cantidades importantes de fondos, indicando muy baja aplicación de esta actividad representando alta debilidad, según el baremo diseñado. Mientras el 40,91\% opinó que siempre y casi siempre se emiten deuda a través de bonos para financiar proyectos de inversión, arribando esta actividad a la categoría de baja aplicación, indicando leve debilidad en referencia al aspecto medido.

Lo obtenido no alcanza a validar lo postulado por Madura (2009), para quien los bonos son instrumentos de deuda a largo plazo que las empresas y el gobierno nacional utilizan para recaudar cantidades importantes de fondos, a menudo lo hacen a partir de un grupo diversificado de prestamistas. De manera similar, no son congruentes con lo Gómez (2010), cuando afirma que los bonos son certificados que se emiten para obtener recursos, estos indican que la empresa pide prestada cierta cantidad de dinero, es decir, cuando un inversionista compra un bono, le está prestando dinero a un gobierno, un ente territorial, una agencia del estado, una compañía o empresa, o simplemente al prestamista.

Los resultados expuestos, pudieran estar reflejados por la inestabilidad económica que atraviesa Venezuela, que si bien no es objeto de estudio en este trabajo puede ser asumida como una de las causas por las cuales las empresas analizadas no consideran las inversiones en bonos como estrategia de financiamiento a largo plazo, no asumiendo el alto riesgo de asumir la venta de bonos dado el alto nivel de incertidumbre del entorno financiero nacional. 
Desde el punto de vista de la dimensión estrategias de financiamiento en el largo plazo, el valor promedio de frecuencias obtenido de sus indicadores, para la suma de las opciones siempre y casi siempre, fue de 57,95\% ubicándose en el rango 50\% $\leq S+C S<75 \%$, con una interpretación de alta aplicación e implicando leve fortaleza, tal como se muestra en la tabla 10.

Tabla 10. Estrategias de financiamiento en el largo plazo.

\begin{tabular}{|c|c|c|}
\hline INDICADOR & $\mathbf{S}+\mathbf{C s}$ & CATEGORÍAS \\
\hline Préstamos a largo plazo & $34,09 \%$ & Baja aplicación / Leve debilidad \\
\hline Capital propio & $93,18 \%$ & Muy alta aplicación / Alta fortaleza \\
\hline Estrategias de dividendos & $72,73 \%$ & Alta aplicación / Leve fortaleza \\
\hline Bonos & $31,82 \%$ & Baja aplicación / Leve debilidad \\
\hline RESUMEN SUB-DIMENSIÓN & $57,95 \%$ & Alta aplicación / Leve fortaleza \\
\hline
\end{tabular}

Los resultados demostraron que en las empresas estudiadas, los préstamos a largo plazo y los bonos no están siendo considerados como estrategias de financiamiento dado que se les categorizó con baja aplicación, lo cual les confiere leve debilidad en estos aspectos (34,09\% y $31,82 \%$, respectivamente). Mientras que se evidencia muy alta aplicación del capital propio como estrategia para financiarse en el largo plazo $(93,18 \%)$, delineándole alta fortaleza en este sentido. Seguido de este tipo de financiamiento ubicaron las estrategias de dividendos, con categoría de alta aplicación implicando una leve fortaleza (72,73\%) para las empresas.

Lo precedente, valida a Gitman (2007), para quien el financiamiento a largo plazo es aquel con un vencimiento inicial de un año o más. Deuda a plazo, utilidades retenidas, arrendamiento operativo y financiero así como los instrumentos financieros bursátiles, representan fuentes de financiamiento de largo plazo, tanto internas como externas, para las empresas en la búsqueda de recursos para financiar sus operaciones.

De igual manera, se valida lo expresado por Block y Hirt (2008), cuando afirman que muchas empresas requieren de financiamientos de sus actividades u operaciones a largo plazo, y, para protegerse contra el peligro de no ser capaz de proporcionar un financiamiento adecuado a corto plazo en periodos de dinero escaso, el administrador puede basarse en fondos a largo plazo para cubrir algunas necesidades a corto plazo.

Con base a lo mostrado para esta dimensión, en las empresas del sector petroquímico, las estrategias de financiamiento en el largo plazo de mayor aplicación son aquellas provenientes de fuentes internas (propias) representadas por el capital propio y las estrategias de dividendos, mientras que consideran baja aplicación a las provenientes de fuentes externas: préstamos a largo plazo y emisión de bonos.

Ahora bien, mostrados los resultados para las dos dimensiones que permitieron el análisis de las estrategias de financiamiento, se presenta el resumen en la tabla 11. Se observa que las estrategias de financiamiento, como eje clave de las estrategias financieras que aplican las 
empresas del sector petroquímico, arriban a una muy alta aplicación $(75,85 \%)$ confiriéndole a estas empresas una alta fortaleza en su gestión financiera.

Tabla 11. Estrategias de financiamiento.

\begin{tabular}{rrl}
\hline DIMENSIÓN & S + Cs & CATEGORÍAS \\
\hline Estrategias de financiamiento en el corto plazo & $93,75 \%$ & Muy alta aplicación / Alta fortaleza \\
Estrategias de financiamiento en el largo plazo & $57,95 \%$ & Alta aplicación / Leve fortaleza \\
\hline RESUMEN DIMENSIÓN & $\mathbf{7 5 , 8 5 \%}$ & Muy alta aplicación / Alta fortaleza \\
\hline
\end{tabular}

Al detalle se puede apreciar que las estrategias para financiarse en el corto plazo ostentan muy alta aplicación $(93,75 \%)$, delineándole una alta fortaleza a las empresas bajo estudio. Mientras que las estrategias de financiamiento en el largo plazo arriban a la categoría de alta aplicación (57,95\%), lo cual se constituye en una leve fortaleza.

Las situaciones descritas validan lo postulado por Ortiz (2005), para quien el término estrategia de financiamiento consiste en la determinación de la mezcla óptima de las fuentes de financiamiento, con las cuales respaldar la operación cotidiana de las empresas y apoyar la ejecución de los planes de negocio, representa un campo complejo que debe encarar la dirección. La dificultad en la fijación de dicha mezcla radica en aceptar que cada alternativa tiene implicaciones en los objetivos trazados, en materia de costos de capital, de liquidez y rentabilidad.

Asimismo, son congruentes por lo expuesto por Block y Hirt (2008), quienes expresan que una decisión crucial la representa la elección del tipo de financiamiento (a corto y largo plazo); la elección entre las dos formas de financiamiento involucra un análisis de flexibilidad de las deudas a corto plazo, de los costos de las deudas a corto plazo en comparación con las de largo plazo y de los riesgos en la rentabilidad.

En este aspecto, se evidenció que en las empresas del sector petroquímico, las formas de financiamiento a corto plazo son las que ocupan mayor peso dentro de las estrategias financieras que optan para la obtención de sus recursos operativos así como de inversión. Adicional a esto, se pudo detectar que en las estrategias de financiamiento en el largo plazo son consideradas las fuentes internas como base de este tipo de financiamiento.

Esta situación es entendible, apoyando esta afirmación en lo manejado en la teoría, puesto que se puede considerar que las decisiones de financiamiento son decisiones cruciales para la empresa, y la preocupación fundamental del gerente financiero es determinar la mejor mezcla de financiamiento; de igual manera, los autores refieren que las posibilidades de obtener financiamiento dependerán en buena medida de la situación del mercado y de la capacidad de gestión de la empresa ante las otras organizaciones que tratan con ella: proveedores y propios clientes. 
Los resultados alcanzan alta congruencia con lo manifestado por Santoma (2008), quien considera que el flujo de cobros de la empresa es una función vital para la buena marcha de todo negocio, ya que es el que aporta el disponible necesario para que la empresa pueda hacer frente a sus propias obligaciones. Asimismo, es la fase donde la empresa realiza sus beneficios, puesto que hasta que la venta no está cobrada no se puede considerar cerrada la transacción comercial.

Asimismo, se logra validar, en alta medida, cuando indica que el proceso de cobro es el generador de liquidez para la empresa y lo que permite su buen funcionamiento, por ello la misión del tesorero debe centrarse en revisar continuamente todos los pasos del circuito de cobro, con el ánimo de reducir, en lo posible, el número de días en cada etapa del proceso, para así reducir el plazo final y real de cobro. En este punto, se tiene que tener siempre presente que el flujo de cobros proporciona el elemento vital (dinero) para que la entidad funcione.

\section{CONCLUSIONES}

$\mathbf{S}$ e identificaron las estrategias de financiamiento aplicadas en las empresas del sector petroquímico, donde se pudo constatar que las empresas bajo estudio muestran muy alta aplicación en sus estrategias de financiamiento en el corto y, alta aplicación de las de largo plazo.; otorgándoles una alta y leve fortaleza en los aspectos medidos.

Se evidenció en cuanto a la aplicación de las estrategias de financiamiento en el corto plazo, muy alta aplicación de éstas siendo una alta fortaleza, al tiempo que todos los indicadores analizados: crédito comercial o crédito de proveedores, línea de crédito a corto plazo, financiamiento de cuentas por cobrar y financiamiento por inventarios arribaron a la categoría de muy alta aplicación, confiriéndole a estas empresas altas fortalezas en estas estrategias aplicadas.

En lo que respecta a las estrategias de financiamiento en el largo plazo, se evidenció alta aplicación por ende una leve fortaleza en este sentido. Es de hacer notar que en las empresas existe mayor aplicación de las estrategias de financiamiento en el largo plazo provenientes de fuentes internas (propias) representadas por el capital propio y las estrategias de dividendos, mientras que consideran baja aplicación a las provenientes de fuentes externas: préstamos a largo plazo y emisión de bonos.

Cabe resaltar, que las empresas pueden diseñar su propio esquema de financiamiento y llegar a crear instrumentos particulares adaptados a sus necesidades, por lo tanto, su financiamiento a largo plazo puede provenir de accionistas (propietarios) o de terceros, según consideren pertinente. 


\section{REFERENCIAS}

Block, S. y Hirt, G. (2008). Fundamentos de administración financiera. 14ava edición. Editorial McGraw-Hill Interamericana, S.A. México

Brachfield, J. (2009). Gestión del crédito y cobro. Claves para prevenir la morosidad y recuperar los impagados. Editorial Profit. Barcelona

Chandler S. (2010). Implantación de la estrategia. Segunda edición. Editorial Mc Graw-Hill. España

Francés, A. (2006). Estrategia y planes para la empresa: con el cuadro de mando integral. Primera Edición. Editorial Pearson Educación. Estado de México. México

Gitman, L. (2007). Principios de Administración financiera. 11ava edición. Editorial Pearson Educación. México

Gómez, A. (2010). Dirección financiera, planificación, gestión y control. Tercera edición. Editorial Financial-Times Prentice Hall. Madrid. España
Madura J. (2009). Administración financiera. Cengage Learning Editores, S.A. México

Ortiz, A. (2005). Gerencia financiera y diagnostico estratégico. Editorial McGraw-Hill. Bogotá. Colombia

Ross, S.; Westerfield, R. y Jeff, J. (2006). Finanzas corporativas. Séptima Edición. Editorial McGrawHill. México

Santoma J. (2008). Gestión de Tesorería. Segunda edición. Ediciones Gestión 2000, S.A. Barcelona, España

Van Horne, J. y Wachowicz, J. (2002). Administración financiera. Editorial Prentice Hall. México

Vera, M. (2009). Estrategias Financieras Empresariales. Guía de estudio para la asignatura. Universidad del Zulia. Maestría de Gerencia de Empresas. Mención Gerencia Financiera. Maracaibo. Venezuela 